PROCEEDINGS OF THE

AMERICAN MATHEMATICAL SOCIETY

Volume 127, Number 6, Pages 1873-1876

S 0002-9939(99)04682-1

Article electronically published on February 17, 1999

\title{
HOMOGENEITY AND THE DISJOINT ARCS PROPERTY
}

\author{
PAWEE KRUPSKI \\ (Communicated by Alan Dow) \\ Dedicated to my wife Ewa
}

\begin{abstract}
Some previous results of the author towards a classification of homogeneous metric continua are improved. The disjoint arcs property is fully revealed in this context. In particular, closed $n$-manifolds, $n=1,2$, are characterized as those homogeneous continua which do not have the disjoint arcs property.
\end{abstract}

\section{INTRODUCTION}

All spaces in this paper are metric separable and all mappings are continuous. A space $X$ is said to be homogeneous, if for any two points $x, y \in X$ there exists a homeomorphism $h: X \rightarrow Y$ such that $h(x)=y$. A space $(X, \rho)$ has the disjoint arcs property $(D A P)$, if for each $\epsilon>0$ and for any two mappings $f, g: I=$ $[0,1] \rightarrow X$ there exist mappings $f^{\prime}, g^{\prime}: I \rightarrow X$ satisfying $f^{\prime}(I) \cap g^{\prime}(I)=\emptyset$ and $\hat{\rho}\left(f, f^{\prime}\right)<\epsilon, \hat{\rho}\left(g, g^{\prime}\right)<\epsilon$, where $\hat{\rho}$ denotes the sup-metric induced by $\rho$.

Relationships between homogeneity and the disjoint arcs property were studied in a series of papers [3]-[5]. It was observed in [3] that all homogeneous locally compact, locally connected spaces of dimension greater than 2 have the DAP. In [4] special attention was paid to homogeneous curves which were proven to have the DAP, except for a simple closed curve. It turned out in [5] that all homogeneous locally connected continua, with the exception of $n$-manifolds, $n=1,2$, have the DAP. There exist interesting examples of 2-dimensional locally connected homogeneous continua different from surfaces. We know only one such continuum in $R^{3}$ described, e.g., in [2] or [6], and called the Pontryagin sphere (because its construction is similar to the original one of the Pontryagin disc in $R^{4}$ ).

In the present paper we prove, among other things, that, actually, all homogeneous continua with the exception of $n$-manifolds, $n=1,2$, have the DAP.

Let us recall four propositions which will be used or strengthened later in this paper.

Proposition $1.1([1,4])$. Suppose $X$ is a one-dimensional locally compact locally connected space with no local separating points. Then either $X$ is a Menger curve

Received by the editors December 3, 1996 and, in revised form, September 25, 1997.

1991 Mathematics Subject Classification. Primary 54F15, 54F65, 57N05.

Key words and phrases. Homogeneous continuum, disjoint arcs property, two-manifold, solenoid, Sierpiński universal curve.

This paper was presented at the 8th Prague Topological Symposium in August 1996.

(C)1999 American Mathematical Society 
manifold or else $X$ contains an open nonempty subset homeomorphic to an open subset of the Sierpiński universal planar curve.

In what follows a solenoid is an inverse limit of a sequence of simple closed curves with covering bonding maps.

Proposition $1.2([4])$. If $X$ is a homogeneous continuum, then one of the following four cases holds.

(i) $X$ is a solenoid.

(ii) $X$ is locally connected and not a simple closed curve.

(iii) There exists an $\alpha>0$ such that each nonempty open subset of $X$ of diameter less than $\alpha$ consists of uncountably many nowhere dense components which are locally connected and have no local separating points.

(iv) Each component $C$ of an arbitrary nonempty open subset of $X$ has an open cover by subsets of $C$ consisting of uncountably many components that are nowhere dense in $C$.

If, additionally, $X$ is 1-dimensional, then $X$ is the Menger universal curve in case (ii), while in case (iii) the components are Menger curve manifolds.

Proposition $1.3([5])$. Let $X$ be a locally connected continuum. Then the following statements are equivalent.

(i) $X$ has no local separating points and no open nonempty subset of $X$ is planar.

(ii) $X$ has the DAP.

Proposition $1.4([5])$. If $X$ is a locally connected homogeneous continuum, then $X$ is not an $n$-manifold, $n=1,2$, if and only if $X$ has the DAP.

\section{Results}

We need an extension of Proposition 1.3 to locally compact spaces.

Proposition 2.1. Let $X$ be a connected, locally connected, locally compact space. Then the following statements are equivalent.

(i) $X$ has no local separating points and no nonempty open subset of $X$ is planar.

(ii) $X$ has the DAP.

Proof. If $X$ is compact, then the proposition is covered by Proposition 1.3. Assume $X$ is not compact. Let $X^{\prime}=X \cup\{\omega\}$ be a one-point compactification of $X$ contained in the Hilbert cube $Q$. Let $f, g: I \rightarrow X$ be two mappings with intersecting images. Take an open connected neighborhood $U$ of $\omega$ in $Q$ such that its closure $\operatorname{cl} U$ is homeomorphic to $Q$ and is disjoint with the continuum $f(I) \cup g(I)$. Observe that the space $Y=X^{\prime} \cup \operatorname{cl} U$ is a locally connected continuum with no local separating points and no planar open nonempty subsets. It follows from Proposition 1.3 that $Y$ has the DAP. If $f^{\prime}, g^{\prime}: I \rightarrow Y$ are two mappings with disjoint images which are close enough to $f$ and $g$, then

$$
f^{\prime}(I) \cup g^{\prime}(I) \subset X .
$$

This shows the DAP for $X$.

The proof of implication (ii) $\Rightarrow$ (i) is the same as the corresponding proof of Proposition 1.3 (see [5, pp. 84-85]). 
Proposition 2.2. Suppose $X$ is a nondegenerate, connected, locally connected, locally compact space with no local separating points, containing no open subset homeomorphic to an open 2-disk. Then either

(1) X contains a nonempty open subset homeomorphic to an open subset of the Sierpinski universal planar curve or

(2) $X$ has the DAP.

Proof. Suppose case (1) does not hold. Observe that $X$ does not contain a nonempty open planar subset. Indeed, if $U \subset X$ were such a set, then $\operatorname{dim} U=1$, because there is no 2-disk in $U$, and, by Proposition 1.1, $U$ would contain a nonempty open subset homeomorphic to an open subset of the Sierpiński curve.

By Proposition 2.1, $X$ has the DAP.

Theorem 2.3. If $X$ is a homogeneous continuum, then one of the following six cases holds.

(1) $X$ is a solenoid.

(2) $X$ is a closed 2-manifold.

(3) $X$ is locally connected and has the DAP.

(4) There exists an $\alpha>0$ such that each nonempty open subset of $X$ of diameter less than $\alpha$ consists of uncountably many nowhere dense components which are 2-manifolds.

(5) There exists an $\alpha>0$ such that each nonempty open subset of $X$ of diameter less than $\alpha$ consists of uncountably many nowhere dense components which are locally connected and have the DAP.

(6) Each component $C$ of an arbitrary nonempty open subset of $X$ has an open cover by subsets of $C$ consisting of uncountably many components that are nowhere dense in $C$.

Proof. In view of Proposition 1.2, it remains to split its case (ii) into (2) and (3), and (iii) into (4) and (5).

If (ii) is satisfied and $X$ is not a 2-manifold, then it has the DAP by Proposition 1.4 .

Assume case (iii) holds. Let $C$ be a component of a nonempty open subset $U$ of $X$ of diameter less than $\alpha$. If $C$ contains an open 2-disk as its open subset, then, since $X$ is homogeneous and components of $U$ are locally connected, every component of $U$ is a 2-manifold and (4) is satisfied. If $C$ contains no open subset homeomorphic to an open 2-disk, then $X$ has the DAP, because otherwise, by Proposition 2.2 and the homogeneity of $X, C$ and all other components of $U$ would be 1-dimensional, so $X$ would be 1-dimensional. This is impossible, in view of Proposition 1.2.

The next result extends [4, Theorem 2.1] to arbitrary homogeneous continua different from solenoids and from 2-manifolds.

Theorem 2.4. If $X$ is a homogeneous continuum which is not a solenoid nor a 2-manifold, then either each component of an arbitrary open subset of $X$ has the DAP or case (4) of Theorem 2.3 holds and $X$ has the DAP.

Proof. We consider all possible cases of Theorem 2.3.

First suppose case (3) holds. Then each component $C$ of an arbitrary open subset of $X$ is an open subset of $X$ and the DAP for $C$ easily follows from the DAP for $X$. 
Suppose case (5) is satisfied. Let $C$ be a component of an open subset $U$ of $X$. If $f, g: I \rightarrow C$ are two mappings with intersecting images, then one can cover the locally connected continuum $A=f(I) \cup g(I)$ by open subsets $G_{1}, \ldots, G_{n}$ of $U$ such that $\operatorname{diam} G_{i}<\alpha$ and $G_{i} \cap A$ is connected for $i=1, \ldots, n$. Denote by $D_{i}$ the component of $G_{i}$ containing $G_{i} \cap A$. Observe that the subset $D=D_{1} \cup \cdots \cup D_{n}$ of $C$ is connected, locally connected and locally compact. In order to verify that $D$ has the DAP, it is convenient to use Proposition 2.1, since each $D_{i}$, and thus $D$, has no local separating point and no open nonempty planar subsets. Hence $C$ also has the DAP.

Case (6) can be treated in the same way as case (3) in the proof of $[4$, Theorem 2.1 , and then we again get the DAP for any component of an arbitrary open subset $U$ of $X$.

Finally, if (4) is satisfied, then $X$ has the DAP by the same argument as in the preceding paragraph, restricted to $U=X$.

The following interesting characterization is an immediate corollary to Theorem 2.4. and it extends [5, Theorem 2] to arbitrary continua.

Theorem 2.5. Let $n=1,2$. A continuum $X$ is a closed $n$-manifold if and only if $X$ is homogeneous and does not have the DAP.

\section{REFERENCES}

1. R. D. Anderson, One-dimensional continuous curves and a homogeneity theorem, Ann. of Math. 68 (1958), 1-16. MR 20:2676

2. W. Jakobsche, Homogeneous cohomology manifolds which are inverse limits, Fund. Math. 137 (1991), 81-95. MR 92i:57019

3. P. Krupski, Recent results on homogeneous curves and ANR's, Topology Proc. 8 (1991), 109-118. MR 94b:54098

4. MR 96a: 54049

5. P. Krupski and H. Patkowska, Menger curves in Peano continua, Colloq. Math. 70 (1996), 79-86. MR 97k:54027

6. W. J. R. Mitchell, D. Repovš and E. V. Ščepin, On 1-cycles and the finite dimensionality of homology 4-manifolds, Topology 31 (1992), 605-623. MR 93f:57024

Mathematical Institute, University of Wroceaw, Pl. Grunwaldzki 2/4, 50-384 WroCŁaW, Poland

E-mail address: krupski@math.uni.wroc.pl 